\title{
ARTICLE
}

\section{Upgrading of shielding for rare decay search in CANDLES}

Kyohei Nakajima ${ }^{\mathrm{a}^{*}}$, Temuge Batpurev ${ }^{\mathrm{b}}$, Wei M. Chan ${ }^{\mathrm{b}}$, Fumiya Dokaku ${ }^{\mathrm{a}}$, Kenichi Fushimic, Kazuki Kanagawa ${ }^{\mathrm{b}}$, Seiya Katagiri ${ }^{\mathrm{b}}$, Kohei Kawasaki ${ }^{\mathrm{a}}$, Bui T. Khai ${ }^{\mathrm{b}}$, Hidetoshi Kino ${ }^{\mathrm{b}}$, Enki Kinoshita ${ }^{\mathrm{b}}$, Tadafumi Kishimoto ${ }^{\text {d,b }}$, Ryuta Hazama ${ }^{\mathrm{e}}$, Hirokazu Hiraoka ${ }^{\mathrm{a}}$, Daiki Hiyama ${ }^{\mathrm{a}}$, Takashi Iida ${ }^{\mathrm{f}}$, Masahiro Ishikawa ${ }^{\mathrm{b}}$, Xiaolong Li ${ }^{\mathrm{b}}$, Tsuyoshi Maeda ${ }^{\mathrm{b}}$, Kenji Matsuoka ${ }^{\mathrm{d}}$, Michael Moser ${ }^{\mathrm{b}}$, Masaharu Nomachid, Izumi Ogawa ${ }^{\mathrm{a}}$, Takaki Ohata ${ }^{\mathrm{b}}$, Hiroyoshi Sato ${ }^{\mathrm{a}}$,

Kazuki Shamoto ${ }^{\mathrm{a}}$, Makiko Shimada ${ }^{\mathrm{a}}$, Masoumeh Shokati ${ }^{\mathrm{b}}$, Naruki Takahashi ${ }^{\mathrm{a}}$, Yasuhiro Takemoto ${ }^{\mathrm{d}}$, Yukichi Takihira ${ }^{\mathrm{d}}$, Yoichi Tamagawa $^{\mathrm{a}}$, Masashi Tozawa ${ }^{\mathrm{a}}$, Kanau Teranishi ${ }^{\mathrm{a}}$, Konosuke Tetsuno ${ }^{\mathrm{b}}$, Van T.T. Trang ${ }^{\mathrm{b}}$

Masahito Tsuzuki ${ }^{\mathrm{b}}$, Saori Umehara ${ }^{\mathrm{d}}$, Wei Wang ${ }^{\mathrm{b}}$, Sei Yoshida ${ }^{\mathrm{b}}$ and Naoki Yotsunaga ${ }^{\mathrm{b}}$

${ }^{a}$ Graduate School of Engineering, University of Fukui, 3-9-1, Bunkyo, Fukui, Fukui 910-8507, Japan; ${ }^{b}$ Graduate School of Science, Osaka University, 1-1, Machikaneyama, Toyonaka, Osaka 560-0043, Japan; ${ }^{c}$ Faculty of Integrated Arts and Science, Tokushima

University, 2-24, Shinkaura-cho, Tokushima 770-8502, Japan; 'Research Center for Nuclear Physics, Osaka University, 10-1, Mihogaoka, Ibaraki, Osaka 567-0047, Japan; ${ }^{e}$ Faculty of Human Environment, Osaka Sangyo University, 3-1-1, Nakagaito, Daito, Osaka, 574-8530, Japan; ${ }^{f}$ Center for Integrated Research in Fundamental Science and Engineering (CiRfSE), Faculty of Pure and Applied Sciences, University of Tsukuba, 1-1-1, Tennodai, Tsukuba, Ibaraki 305-8571, Japan

In the CANDLES experiment aiming to search for the very rare neutrino-less double beta decays $(0 \mathrm{v} \beta \beta)$ using ${ }^{48} \mathrm{Ca}$, we introduced a new shielding system for high energy $\gamma$-rays from neutron captures in massive materials near the detector, in addition to the background reduction for ${ }^{232} \mathrm{Th}$ decays in the $0 v \beta \beta$ target of $\mathrm{CaF}_{2}$ crystals. The method of background reduction and the performance of newly installed shielding system are described.

\section{Keywords: double beta decay; underground experiment; low background measurement; $\gamma$-rays from} neutron captures

\section{Introduction}

The neutrino-less double beta decay $(0 v \beta \beta)$ is a process beyond the standard model and has not been observed yet. The existence of $0 v \beta \beta$ leads to three important facts; (1) The lepton number violation, which is a key of our matter dominated universe. (2) The Majorana nature of neutrinos, which indicates that particle is its own anti-particle. (3) The absolute scale of neutrino mass. A mass hierarchy of neutrinos is determined by the $0 v \beta \beta$ observation.

In $0 v \beta \beta$, two electrons are emitted and the $0 v \beta \beta$ measurement tries to find the signals at sum energies of electrons corresponding to the $Q$ value. The $0 v \beta \beta$ is a quite rare phenomenon and low background techniques are required to observe such rare decays. Until now, only a lower limit of half-life was experimentally obtained to $10^{26}$ year by the KamLAND experiment using ${ }^{136} \mathrm{Xe}[1]$.

CANDLES (CAlcium fluoride for the study of Neutrinos and Dark matters by Low Energy Spectrometer) is a double beta decay experiment using ${ }^{48} \mathrm{Ca}$ in $\mathrm{CaF}_{2}$ crystals [2]. The advantage of ${ }^{48} \mathrm{Ca}$ is the highest $\mathrm{Q}$ value among all isotope candidates $(4.3 \mathrm{MeV})$, and therefore the background sources are limited in such

*Corresponding author. Email: nkyohei@u-fukui.ac.jp a high energy region. The disadvantage of ${ }^{48} \mathrm{Ca}$ is a low natural abundance of $\sim 0.2 \%$, and the enrichment technique is under development [3].

The best result thus far obtained for a lower limit of half-life using ${ }^{48} \mathrm{Ca}$ is $5.8 \times 10^{22}$ year $(90 \%$ Confidence Level (C.L.)) by the ELEGANT VI experiment [4]. It was found that reduction of $\gamma$-ray background due to neutron capture around the detector is indispensable to improve this limit (section 3). This paper describes establishing a new shield system for the purpose.

\section{Detector}

The CANDLES detector is located in the Kamioka underground laboratory (Gifu Pref., Japan) where the overburden is $2,700 \mathrm{~m}$.w.e. (meter water equivalent). The muon flux is about $10^{-5}$ of the ground level, and it leads to the lower muon-related environmental radiations such as neutrons.

A schematic view of the detector is shown in Figure 1. We use $96 \mathrm{CaF}_{2}$ crystals $\left(10^{3} \mathrm{~cm}^{3}\right)$ which contain $305 \mathrm{~g}$ of ${ }^{48} \mathrm{Ca}$, as the scintillator detector and the $0 v \beta \beta$ target. The scintillation light from $\mathrm{CaF}_{2}$ crystals are observed by 62 photomultiplier tubes (PMTs) with the light pipe system which has a $93 \%$ reflectivity. The $\mathrm{CaF}_{2}$ crystals are immersed in the liquid scintillator (LS) which works as active veto, and an acrylic tank containing LS is set in 
the water passive shield with an average thickness of $\sim 1$ $\mathrm{m}$. The whole system is installed in a stainless steel tank with $3 \mathrm{~m}$ diameter and $4 \mathrm{~m}$ height.
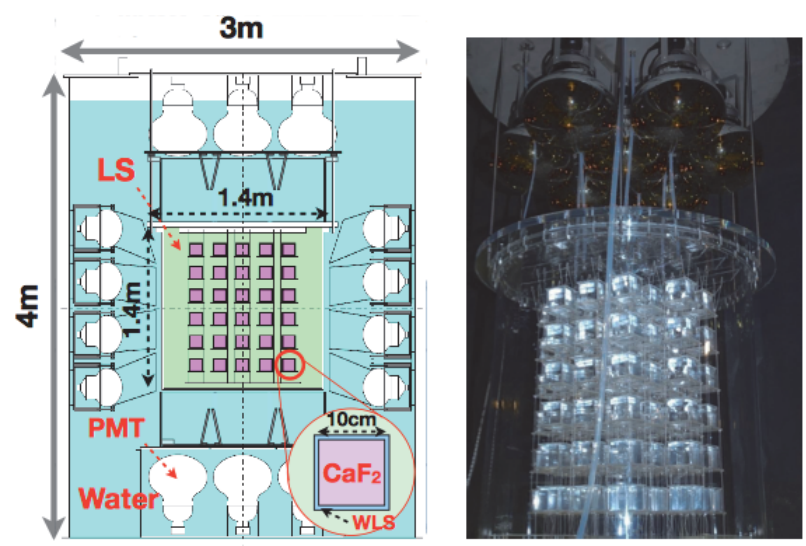

Figure 1. A schematic view of the detector. Left: A drawing of the detector from the side view. The whole detector is installed in a cylindrical stainless steel tank. Right: A photo of $96 \mathrm{CaF}_{2}$ crystals which are immersed in LS.

A distinction of the experiment is a $4 \pi$ active shield by LS. The time constant of the pulse shapes are $\sim 1 \mu$ sec and $\sim 10$ nsec for $\mathrm{CaF}_{2}$ and $\mathrm{LS}$, respectively. When external $\gamma$-rays react with LS, the observed pulse shapes are different from that of the signal (i.e. the pulse of $\beta$ rays in $\mathrm{CaF}_{2}$ ). By using this pulse shape difference, events which contain the LS pulse are rejected event by event by analyzing the pulse-shape data which are recorded by a $500 \mathrm{MHz}$ FADC (Flash ADC) (LS cut). This active system is also useful for rejection of internal $\beta$ decays in $\mathrm{CaF}_{2}$ when emitted $\gamma$-rays deposit their energy in LS.

\section{Background}

\subsection{Background candidates}

The background sources are suppressed thanks to the high $\mathrm{Q}$ value of ${ }^{48} \mathrm{Ca}$. There are three background candidates around 4.3 MeV. As shown in Figure 2, two of them are decay chain of a radioactive impurity, ${ }^{232} \mathrm{Th}$ in $\mathrm{CaF}_{2}$ crystals; (1) ${ }^{208} \mathrm{Tl}\left(\beta\right.$ decay, $\left.\mathrm{Q}_{\beta}=5.0 \mathrm{MeV}\right),(2)$ Pile-up events of ${ }^{212} \mathrm{Bi}$ and ${ }^{212} \mathrm{Po}$ (Maximum energy is $5.3 \mathrm{MeV})$ due to short half-life of ${ }^{212} \mathrm{Po}(\sim 300 \mathrm{~ns})$. Since the time window of the pulse shape is $4 \mu \mathrm{sec}$, the signals due to ${ }^{212} \mathrm{Bi}$ and ${ }^{212} \mathrm{Po}$ decays are observed as a single pulse.

The third background is high energy $\gamma$-rays from neutron captures in massive materials near the detector, called as $(n, \gamma)$ background. Despite of the measurement in low neutron flux and with the combination of the LS active shield and the water shield, the $(n, \gamma)$ events proved the largest background as mentioned in section 3.3.

\section{2. ${ }^{208} \mathrm{Tl}$ and ${ }^{212} \mathrm{Bi}^{212}$ Po backgrounds}

We employed pure $\mathrm{CaF}_{2}$ crystals developed by choosing material powders which has a less radioactivity before growing the crystals [2,3]. The average ${ }^{232} \mathrm{Th}$ activity of 96 crystals is $\sim 29 \mu \mathrm{Bq} / \mathrm{kg}$ and 27 of them have an activity below $10 \mu \mathrm{Bq} / \mathrm{kg}$.

The backgrounds related to ${ }^{232} \mathrm{Th}$-decay series can be removed through the off-line data analysis (analysis cut) [4]. Since the decay of ${ }^{212} \mathrm{Bi}$ ( $\beta$ decay) and ${ }^{212} \mathrm{Po}(\alpha$ decay) are observed as a single event due to the short half-life of ${ }^{212} \mathrm{Po}$ (300 ns), such events are rejected if time differences between ${ }^{212} \mathrm{Bi}$ and ${ }^{212} \mathrm{Po}$ are longer than $20 \mathrm{nsec}$, which is a limit to distinguish the double pulse. If time differences are shorter than $20 \mathrm{nsec}, \sim 95 \%$ events can be rejected by using the pulse shape difference between $\alpha$ particles and $\beta$ particles in $\mathrm{CaF}_{2}$ [5]. The total rejection efficiency of this "Bi-Po cut" for ${ }^{212} \mathrm{Bi}^{212} \mathrm{Po}$ background is about $99 \%$.

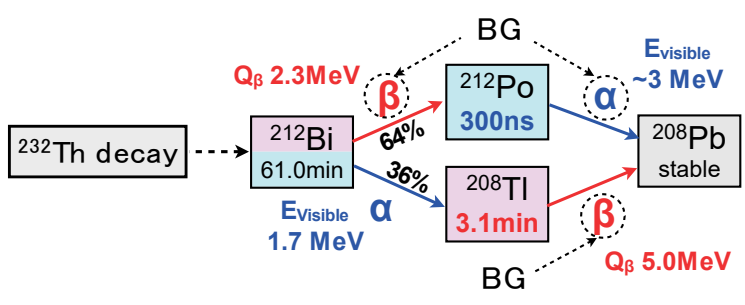

Figure 2. A schematic diagram of the ${ }^{208} \mathrm{Tl}$ and ${ }^{212} \mathrm{Bi}^{212} \mathrm{Po}$ backgrounds in ${ }^{232} \mathrm{Th}$ decay chain.

To reject the ${ }^{208} \mathrm{Tl}$ background, we apply veto time to the crystal where $\alpha$ particles from a parent nuclei ${ }^{212} \mathrm{Bi}$ are observed at $1.7 \mathrm{MeV}$ (Figure 2). The veto time window is 12 minutes which is long enough compared to the ${ }^{208} \mathrm{Tl}$ half-life, 3.1 minutes to inhibit collection of event due to the decay of ${ }^{208} \mathrm{Tl}$. The rejection efficiency of this " ${ }^{208} \mathrm{Tl}$ cut" is evaluated to be $\sim 90 \%$. In order to achieve better rejection efficiency, it is necessary to apply veto time also for crystals neighboring to that where the $\alpha$-particles are observed although it causes larger dead time of the measurement time. It is because the position of the crystal is sometimes misidentified due to the interaction of successive $\gamma$-rays from daughter nucleus ${ }^{208} \mathrm{Tl}$ with neighboring crystals. We call this cut as "multi crystal veto".

\section{3. $(n, \gamma)$ background}

Even above Q value, nonnegligible amounts of events were observed. Figure 3 shows the observed energy spectrum in the year-2013 data. Many events above Q value were observed even in the energy region from 7 to $8 \mathrm{MeV}$. The cause of these events was identified as high energy $\gamma$-rays from neutron captures in massive material around the detector such as rock and stainless steel. For example, the specific $\gamma$-ray energy from neutron captures on ${ }^{56} \mathrm{Fe}$ is $7.6 \mathrm{MeV}$. These $\gamma$-rays sometimes deposit almost entire energy in $\mathrm{CaF}_{2}$ crystals and then remain as a background.

In order to confirm the origin of this background, we set a ${ }^{252} \mathrm{Cf}$ source outside the stainless steel tank to increase the neutron flux. The observed spectrum with a ${ }^{252} \mathrm{Cf}$ source is consistent with the data of normal run in the high energy region. The energy spectra with a 
neutron source and in normal run were simulated by the Monte Carlo (MC) simulation using the code Geant4 [6]. As a result, the observed spectrum was well reproduced by considering $\gamma$-rays from neutron captures in rock and stainless steel. The $(\mathrm{n}, \gamma)$ background was estimated to be $76 \pm 9$ (statistical.) events/year/96crystals, which is beyond acceptable level.

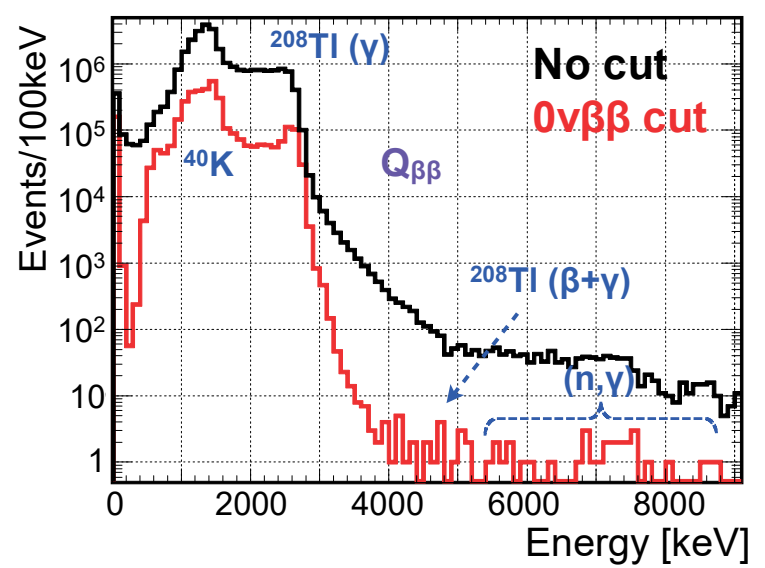

Figure 3. The observed energy spectrum in the year-2013 data. The black line shows the spectrum without analysis cuts, and the red line shows the one with all analysis cuts ( $0 v \beta \beta$ cut). We can see some events above $\mathrm{Q}_{\beta}(4.3 \mathrm{MeV})$. These events were identified as $(\mathrm{n}, \gamma)$ background.

\section{Shield for $(n, \gamma)$ background}

\subsection{Shield design}

Since the $(n, \gamma)$ events proved to be the most serious background in CANDLES, we installed an additional shield in the detector. The goal of the $(n, \gamma)$ background rate is about 1 events/year/96crystals, which is $\sim 1 / 80$ of the event rate in Figure 3. A shield design was optimized by Geant 4 simulation. A schematic view of the design is shown in Figure 4.

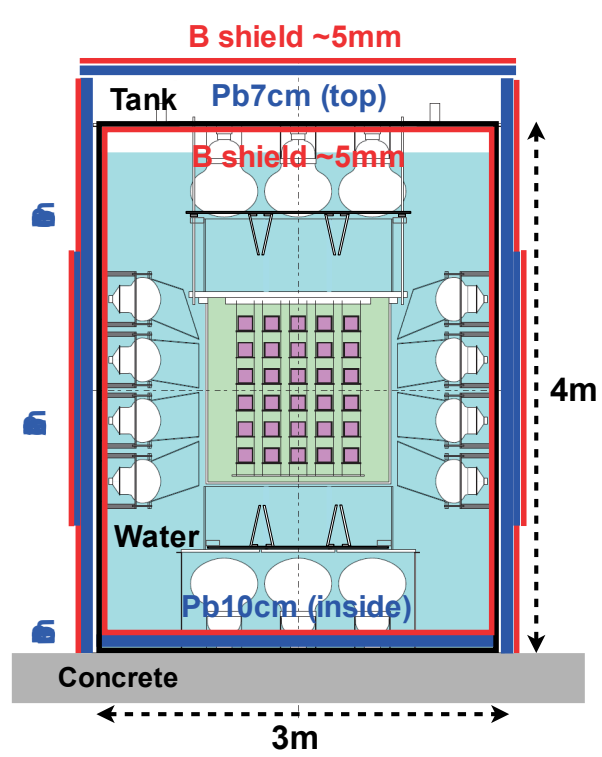

Figure 4. A schematic view of the additional passive shield for the $(\mathrm{n}, \gamma)$ background reduction. The blue and red parts show $\mathrm{Pb}$ shield and $\mathrm{B}$-containing rubber sheet, respectively.
We installed $\mathrm{Pb}$ shields for $\gamma$-rays from neutron captures in rocks, and Si rubber sheets including $40 \mathrm{wt} \%$ of $\mathrm{B}_{4} \mathrm{C}\left(6.2 \mathrm{wt} \%\right.$ of $\left.{ }^{10} \mathrm{~B}\right)$ inside and outside the detector to reduce thermal neutron captures in stainless steel tank. The typical $\mathrm{Pb}$ thickness is about $10 \mathrm{~cm}$ to reduce $\gamma$-rays with several $\mathrm{MeV}$ to $\sim 1 / 100$. Considering the weight limit on the top of the detector, $\mathrm{Pb}$ thickness in the top was suppressed to $7 \mathrm{~cm}$, but $\mathrm{Pb}$ thickness was increased to $12 \mathrm{~cm}$ in the center of the detector side, where thickness of the passive water shield in the detector is thinnest. In the bottom, $\mathrm{Pb}$ shield was set inside the detector, so as to avoid significant modification of the system.

By installing the shield, the $(n, \gamma)$ background rate was expected to be $\sim 0.7( \pm 50 \%)$ events/year/96crystals from the MC simulation. The breakdown of $(n, \gamma)$ background from rocks and tank is $0.34 \pm 0.14$ and $0.4 \pm 0.2$ events/year/96crystals, respectively.

\subsection{Shield construction}

The shield construction started in March 2015. The status of the construction is shown in Figure 5. Firstly, we started to set $\mathrm{Pb}$ shields from the outside of the detector. The bottom $\mathrm{Pb}$ blocks were set inside of the tank. They were covered by the liquefied B shield to block $\mathrm{Pb}$ dissolution to the water.

The $\mathrm{B}_{4} \mathrm{C}$ containing rubber sheets was set after the $\mathrm{Pb}$ construction. They were supported by the punched metal outside and the aluminum frame inside. After setting inner sheets, we poured the liquefied B shield in the bottom of the tank. They were solidified in 1 day.

Finally, we closed the detector after installing $\mathrm{Pb}$ shield on the top. The shield construction finished in March 2016 and then we started the data taking. We checked $\mathrm{B}$ and $\mathrm{Pb}$ elution into water periodically, and no elution has been observed yet.
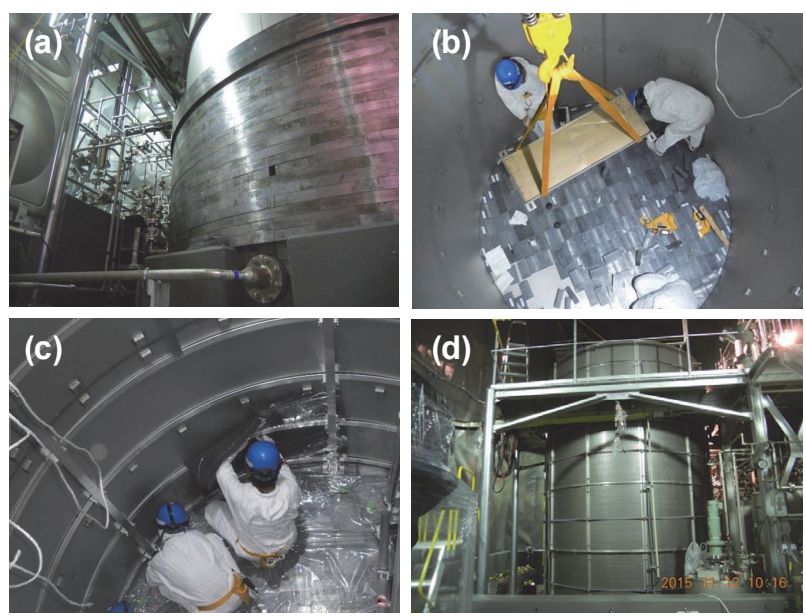

Figure 5. Photos taken in the shield construction. (a) $\mathrm{Pb}$ shield outside the tank. (b) $\mathrm{Pb}$ shield in the bottom of the tank. (c) B sheet set inside the tank. (d) Overview of the shield. 


\section{Results}

\subsection{Energy spectrum after shielding}

The energy spectrum after $(n, \gamma)$ shield setup is shown in Figure 6. In this case, the LS cut and the ${ }^{208} \mathrm{Tl}$ cut are not applied to emphasize counting statistics in the high energy region. The red and green histograms show the spectrum before $(n, \gamma)$ shield setup, and the blue histogram shows the spectrum after setup. All spectra are normalized to the event rate in 56.9 days.

The peaks at $1.46 \mathrm{MeV}$ and at $2.6 \mathrm{MeV}$ caused by environmental $\gamma$-rays from ${ }^{40} \mathrm{~K}$ and ${ }^{208} \mathrm{Tl}$, respectively, are reduced by about one order of magnitude by the additional shield. This would help to reduce the dead time when we apply the ${ }^{208} \mathrm{Tl}$ veto. Events in 3 to $4 \mathrm{MeV}$ region were reduced by replacing the O-ring and $\mathrm{Al}$ sleeve near the $\mathrm{CaF}_{2}$ crystals with ones having less ${ }^{232} \mathrm{Th}$ activity.

The events above $5 \mathrm{MeV}((\mathrm{n}, \gamma)$ background) were clearly reduced by $(\mathrm{n}, \gamma)$ shield setup. The reduction rate is an order of $10^{-2}$ on average, and consistent with the design value in the MC simulation.

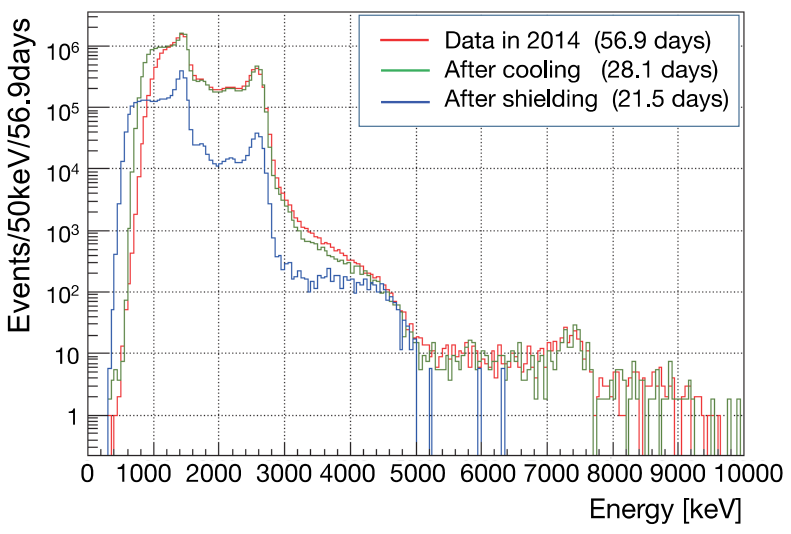

Figure 6. The observed energy spectrum without LS cut and ${ }^{208} \mathrm{Tl}$ cut before and after the $(\mathrm{n}, \gamma)$ shield setup. The red and green histograms show the spectra before setup, and the blue histogram shows the spectrum after setup. All spectra were normalized to the event rate in 56.9 days.

\subsection{Sensitivity of CANDLES after $(n, \gamma)$ shielding setup}

Then, the analysis cuts for $0 v \beta \beta$ selection were applied to simulate realistic situation. The analysis was performed for all crystals or pure crystals whose ${ }^{232} \mathrm{Th}$ activity were below $10 \mu \mathrm{Bq} / \mathrm{kg}$. The energy spectrum for 27 pure crystals is shown in Figure 7. A summary of the analysis result is shown in Table 1.

As a conclusion, no events were observed in the region of interest $\left(\mathrm{Q}_{\beta \beta-1 \sigma}{ }^{+2 \sigma} ; 4170-4480 \mathrm{keV}\right)$ during the measurement for 21.5 days. From the number of expected backgrounds and the signal efficiency, the sensitivity of the present CANDLES for $0 v \beta \beta$ half-life is calculated to be $0.9 \times 10^{23}$ year and $0.5 \times 10^{23}$ year for all crystals and 27 crystals, respectively. Comparing with the best half-life limit using ${ }^{48} \mathrm{Ca}$ so far $\left(0.6 \times 10^{23}\right.$ year), it will be possible to obtain the better result in a 1 year measurement.

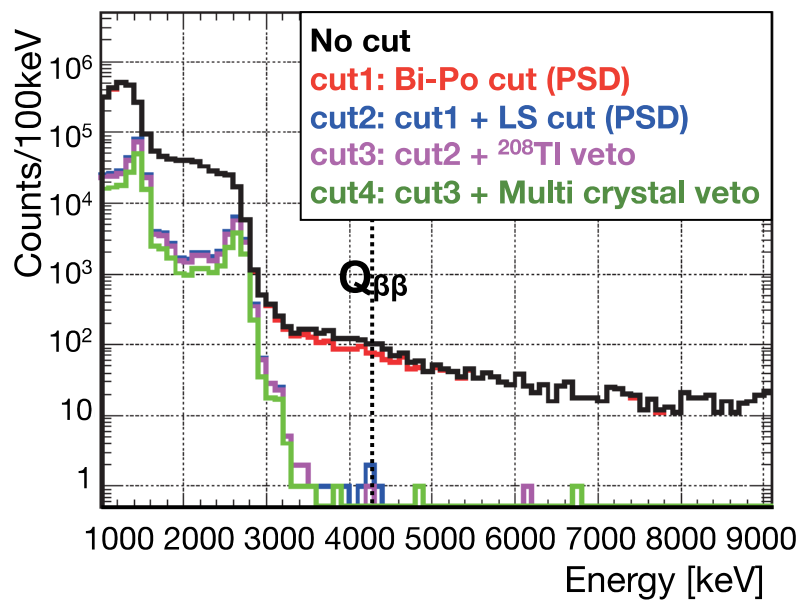

Figure 7. The energy spectrum after $(\mathrm{n}, \gamma)$ shield setup in the 21.5 days live-time measurement by applying various analysis cuts, i.e. the Bi-Po cut, the LS cut, the ${ }^{208} \mathrm{Tl}$ veto, and the multi crystal veto that are described in section $3.2,2,3.3,3.3$, respectively. The green histogram shows the spectrum obtained with all analysis cuts. There are no events in the

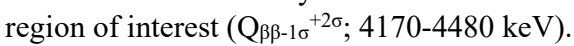

Table 1. Results of with the $0 v \beta \beta$ analysis after shield setup.

\begin{tabular}{ccc}
\hline Crystal selection & All crystals & 27 crystals \\
\hline Number of events & 0 & 0 \\
Expected ${ }^{208} \mathrm{Tl} \mathrm{BG}$ & 1.4 & 0.14 \\
Expected $(\mathrm{n}, \gamma) \mathrm{BG}$ & 0.04 & 0.01 \\
Signal efficiency & 0.30 & 0.30 \\
Sensitivity in 1 year & $0.9 \times 10^{23}$ year & $0.5 \times 10^{23}$ year \\
\hline
\end{tabular}

\section{Conclusion}

CANDLES is designed for the study of double beta decay with ${ }^{48} \mathrm{Ca}$. The main background sources are ${ }^{208} \mathrm{Tl}$ decay in $\mathrm{CaF}_{2}$ crystal and high energy $\gamma$-rays from neutron captures in surrounding rocks and stainless steel. The latter $(\mathrm{n}, \gamma)$ events were found to be the largest background, and we installed an additional passive shield consisting of $\mathrm{Pb}$ and $\mathrm{B}_{4} \mathrm{C}$ rubber sheets. We confirmed that the reduction rate of the $(n, \gamma)$ background was about $10^{-2}$. No events were observed in the region of interest in 21.5 days measurement. The expected sensitivity in 1 year is $0.9 \times 10^{23}$ year, which is the best result among the $0 v \beta \beta$ measurements with ${ }^{48} \mathrm{Ca}$.

\section{References}

[1] A. Gando et al., Phys. Rev. Lett. 117 (2016), 082503.

[2] T. Kishimoto et al., Proc. of 4th Workshop on Neutrino Oscillations and Their Origins, (2003), p.338.

[3] T. Kishimoto et al., Prog. of Theor. Phys. (2015), $033 \mathrm{D} 03$.

[4] S. Umehara et al., Phys. Rev. C 78 (2008), 058501.

[5] S. Umehara et al., Physics Procedia 61 (2015), pp.283-288.

[6] S. Agostinelli et al., Nucl. Instrum. Methods Phys. Res. A 506 (2006), pp.250-303. 